\title{
Coulomb gauge gluon propagator on anisotropic lattices
}

\section{Yoshiyuki Nakagawa*}

Research Institute for Information Science and Education, Hiroshima University, Higashi-Hiroshima, Hiroshima, 739-8521, Japan

E-mail: nkgwercnp.osaka-u.ac.jp

\section{Atsushi Nakamura}

Research Institute for Information Science and Education, Hiroshima University,

Higashi-Hiroshima, Hiroshima, 739-8521, Japan

E-mail: nakamura@riise.hiroshima-u.ac.jp

\section{Takuya Saito}

Integrated Information Center, Kochi University

Kochi, 780-8520, Japan

E-mail: tsaitoulkochi-u.ac.jp

\section{Hiroshi Toki}

Research Center for Nuclear Physics, Osaka University

Ibarakisi, Osaka 567-0044, Japan

E-mail: toki@rcnp.osaka-u.ac.jp

\begin{abstract}
We calculate the transverse and the time-time components of the Coulomb-gauge gluon propagator in SU(3) lattice Yang-Mills theory both on isotropic and anisotropic lattices. The problem of scaling violation observed on the isotropic lattice is drastically reduced as the anisotropy increases; namely, the system approaches the Hamiltonian limit. In the infrared region, the transverse gluon propagator exhibits a turnover and the temporal gluon propagator shows divergent behavior.
\end{abstract}

The XXVII International Symposium on Lattice Field Theory - LAT2009

July 26-31 2009

Peking University, Beijing, China

\footnotetext{
* Speaker.
} 


\section{Introduction}

Coulomb gauge provides a very clear picture of color confinement. Coulomb gauge is a physical gauge in the sense that the color Gauss' law can be formally solved, and only transverse degrees of freedom appears as dynamical degrees of freedom. The striking feature of Coulomb gauge is that an instantaneous interaction shows up in the Hamiltonian, which is requisite for color confinement. In the Gribov-Zwanziger scenario, the path integral is dominated by the configurations near the Gribov horizon where the lowest eigenvalue of the Faddeev-Popov (FP) ghost operator vanishes [1]. This results in an enhancement of the near-zero modes of the ghost operator, and it has been confirmed by the lattice simulations [2,3]. Accordingly, the color-Coulomb instantaneous interaction becomes a confining interaction. The color-Coulomb potential can be obtained by measuring the correlator of the temporal link variable at fixed time, and lattice QCD simulations exhibit that the color-Coulomb potential rises linearly at large distances and its string tension is larger than the string tension of the static Wilson potential [4, 5, 6, 7], which is expected from the Zwanziger's inequality [8]. On the other hand, the color-Coulomb potential has been evaluated by inverting the FP ghost matrix, and it has been shown that the color-Coulomb string tension almost saturates the Wilson string tension [9].

The transverse gluon propagator is expected to be suppressed in the infrared (IR) region due to the proximity of the Gribov region in the IR direction in the Gribov-Zwanziger scenario [10]. The instantaneous transverse gluon propagator has been measured by Monte Carlo simulations $[11,12,13,14]$, and recent studies have revealed that it shows scaling violation [13, 14]; namely, the gluon propagator calculated at different lattice couplings does not fall on top of a single curve after multiplicative renormalization.

In order to circumvent the problem of scaling violation, the authors of [13] have measured the unequal-time gluon propagator

$$
D^{\operatorname{tr}}\left(\vec{p}, p_{4}\right)=\left\langle A\left(\vec{p}, p_{4}\right) A\left(-\vec{p},-p_{4}\right)\right\rangle
$$

and extracted the equal-time propagator $D^{\operatorname{tr}}(|\vec{p}|)$ by eliminating the $p_{4}$ dependence of the unequaltime propagator. It has been concluded that $D^{\operatorname{tr}}(|\vec{p}|)$ is multiplicatively renormalizable in the Hamiltonian limit and well fitted with the Gribov-type form of the propagator. The method was applied only to the transverse gluon propagator and it was not studied for the time-time component of the equal-time gluon propagator if scaling violation can be solved by this procedure.

In [14], a new momentum cut is introduced in addition to the cone cut and the cylinder cut, by which high momentum data that suffer from discretization errors are excluded from the analysis of the instantaneous propagators. It has been shown that this procedure successfully reduces scaling violation for the transverse gluon propagator while it fails for the time-time component of the gluon propagator.

The problem of scaling violation of the instantaneous propagator can be seen even at the tree level on a finite temporal lattice spacing (we refer to a forthcoming paper for an explicit calculation). The reason is that the energy integral does not run from $-\infty$ to $\infty$ but from $-\pi / a_{\tau}$ to $\pi / a_{\tau}$ on a finite lattice, and this introduces the spurious $|\vec{p}|$ dependence on the free equal-time propagator. Therefore, we expect that the instantaneous propagator is multiplicatively renormalizable in 
the Hamiltonian limit $\xi=a_{s} / a_{\tau} \rightarrow \infty$. To make this point clear, we calculate the transverse and temporal components of the instantaneous gluon propagator on anisotropic lattices.

\section{Lattice setup and observables}

The lattice configurations are generated by the heat-bath Monte Carlo technique with the standard Wilson plaquette action,

$$
S=\frac{\beta}{\xi_{B}} \sum_{n, i<j \leq 3} \mathfrak{R e} \operatorname{Tr}\left(1-U_{i j}(n)\right)+\beta \xi_{B} \sum_{n, i \leq 3} \mathfrak{R e} \operatorname{Tr}\left(1-U_{i 4}(n)\right) .
$$

Here $U_{\mu v}(n)$ indicates the plaquette operator, and $\beta=2 N_{c} / g^{2}$ is the lattice coupling. On the isotropic lattice, the bare anisotropy $\xi_{B}$ is 1 and the action can be written in a familiar form

$$
S=\beta \sum_{n, \mu<v} \mathfrak{R e} \operatorname{Tr}\left(1-U_{\mu v}(n)\right)
$$

$\xi_{B}$ differs from the renormalized anisotropy $\xi$ which is defined as the ratio of the spatial lattice spacing to the temporal lattice spacing. The ratio of $\xi_{B}$ and $\xi$ can be determined non-perturbatively by matching the spatial and the temporal Wilson loop on anisotropic lattices. We use the relation obtained by Klassen for the range $1 \leq \xi_{B} \leq 6$ and $5.5 \leq \beta \leq \infty[15]$ :

$$
\frac{\xi}{\xi_{B}}=1+\left(1-\frac{1}{\xi}\right) \frac{\eta(\xi)}{6} \frac{1+a_{1} g^{2}}{1+a_{0} g^{2}} g^{2}
$$

where $a_{0}=-0.77810, a_{1}=-0.55055$, and

$$
\eta(\xi)=\frac{1.002503 \xi_{B}^{3}+0.39100 \xi_{B}^{2}+1.47130 \xi_{B}-0.19231}{\xi_{B}^{3}+0.26287 \xi_{B}^{2}+1.59008 \xi_{B}-0.18224} .
$$

We adopt the values of the lattice spacing given in [16] for $\xi=2$ and in [17] for $\xi=4$, where the static quark potential was measured to set the scale. For the isotropic lattice, the scale is set by using Necco-Sommer scaling relation [18]. In our simulations, the first 5000 sweeps are discarded for thermalization, and we measured the equal-time gluon propagator for $40-100$ configurations, each of which is separated by 100 sweeps. All the lattice parameters are given in Table 1.

In Coulomb gauge the transversality condition

$$
\partial_{i} A_{i}(\vec{x}, t)=0
$$

is imposed on the gauge fields at each time slice, where $i$ runs from 1 to 3 . On a lattice, gauge configurations satisfying Coulomb gauge condition can be obtained by minimizing the functional

$$
F_{U}[g]=\sum_{i=1}^{3} \sum_{\vec{x}} \mathfrak{R e} \operatorname{Tr}\left(1-U_{i}^{g}(\vec{x}, t)\right),
$$

defined on each time slice. Here $U_{i}^{g}(\vec{x}, t)=g(\vec{x}, t) U_{i}(\vec{x}, t) g^{\dagger}(\vec{x}+\hat{i}, t)$ is the gauge-rotated configuration. The functional derivative of Eq. (2.6) with respect to $g$ reproduces the Coulomb gauge condition in the continuum limit with the linear definition of the gauge field,

$$
A_{\mu}^{\mathrm{lat}}(\vec{x}, t)=\left.\frac{U_{\mu}(\vec{x}, t)-U_{\mu}^{\dagger}(\vec{x}, t)}{2 i g a}\right|_{\text {traceless }} .
$$




\begin{tabular}{cccccccc}
\hline \hline$\xi=a_{s} / a_{\tau}$ & $L_{s}^{3} \times L_{\tau}$ & $\beta$ & $\xi_{B}$ & $a_{s}^{-1}[\mathrm{GeV}]$ & $a_{s}[\mathrm{fm}]$ & $V\left[\mathrm{fm}^{4}\right]$ & \# of confs. \\
\hline \hline \multirow{4}{*}{1} & $32^{4}$ & 5.70 & 1 & 1.160 & 0.1702 & $5.45^{4}$ & 100 \\
& $48^{4}$ & $:$ & $:$ & $:$ & $:$ & $8.17^{4}$ & 40 \\
& $32^{4}$ & 5.80 & $:$ & 1.446 & 0.1364 & $4.37^{4}$ & 100 \\
& $32^{4}$ & 6.00 & $:$ & 2.118 & 0.0932 & $2.98^{4}$ & 100 \\
& $48^{4}$ & $:$ & $:$ & $:$ & $:$ & $4.47^{4}$ & 40 \\
$32^{4}$ & 6.20 & $:$ & 2.914 & 0.0677 & $2.17^{4}$ & 100 \\
\hline \multirow{6}{*}{2} & $16^{3} \times 32$ & 5.80 & 1.674 & 1.104 & 0.1787 & $2.86^{4}$ & 100 \\
& $24^{3} \times 48$ & $:$ & $:$ & $:$ & $:$ & $4.29^{4}$ & 100 \\
& $16^{3} \times 32$ & 6.00 & 1.705 & 1.609 & 0.1227 & $1.96^{4}$ & 100 \\
& $24^{3} \times 48$ & $:$ & $:$ & $:$ & $:$ & $2.94^{4}$ & 100 \\
& $16^{3} \times 32$ & 6.10 & 1.718 & 1.889 & 0.1045 & $1.67^{4}$ & 100 \\
& $24^{3} \times 48$ & $:$ & $:$ & $:$ & $:$ & $2.51^{4}$ & 100 \\
\hline & $16^{3} \times 64$ & 5.75 & 3.072 & 1.100 & 0.1794 & $2.87^{4}$ & 50 \\
& $24^{3} \times 96$ & $:$ & $:$ & $:$ & $:$ & $4.31^{4}$ & 50 \\
& $32^{3} \times 128$ & $:$ & $:$ & $:$ & $:$ & $5.74^{4}$ & 50 \\
& $48^{3} \times 192$ & $:$ & $:$ & $:$ & $:$ & $8.61^{4}$ & 50 \\
& $16^{3} \times 64$ & 5.95 & 3.159 & 1.623 & 0.1216 & $1.95^{4}$ & 50 \\
& $24^{3} \times 96$ & $:$ & $:$ & $:$ & $:$ & $2.92^{4}$ & 50 \\
& $32^{3} \times 128$ & $:$ & $:$ & $:$ & $:$ & $3.89^{4}$ & 50 \\
$48^{3} \times 192$ & $:$ & $:$ & $:$ & $:$ & $5.84^{4}$ & 50 \\
$16^{3} \times 64$ & 6.10 & 3.211 & 2.030 & 0.0972 & $1.56^{4}$ & 50 \\
$24^{3} \times 96$ & $:$ & $:$ & $:$ & $:$ & $2.33^{4}$ & 50 \\
& $32^{3} \times 128$ & $:$ & $:$ & $:$ & $:$ & $3.11^{4}$ & 50 \\
& $48^{3} \times 192$ & $:$ & $:$ & $:$ & $:$ & $4.67^{4}$ & 50 \\
\hline \hline
\end{tabular}

Table 1: Simulation parameters to calculate the equal-time gluon propagator.

The Coulomb gauge fixing has been done using iterative method with the Fourier acceleration [19], and the gauge fixing is stopped if $\left(\partial_{i} A_{i}\right)^{2}<10^{-14}$ at each time slice.

We calculate the transverse and time-time component of the equal-time gluon propagator,

$$
D_{\mu \nu}^{a b}(\vec{x}-\vec{y})=\left\langle A_{\mu}^{a}(\vec{x}) A_{v}^{b}(\vec{y})\right\rangle=D_{\mu \nu}^{a b}(\vec{x}-\vec{y}),
$$

in the momentum space,

$$
\begin{aligned}
& D_{i j}^{a b}(\vec{p})=\delta^{a b}\left(\delta_{i j}-\frac{p_{i} p_{j}}{|\vec{p}|^{2}}\right) D^{\operatorname{tr}}(|\vec{p}|) \\
& D_{44}^{a b}(\vec{p})=\delta^{a b} \frac{Z^{44}(|\vec{p}|)}{|\vec{p}|^{2}} .
\end{aligned}
$$

The gauge field is defined as Eq. (2.7). The dressing function $Z^{44}$ is constant for $|\vec{p}|$ at the tree level. In the Gribov-Zwanziger scenario, this is expected to diverge in the IR limit resulting in the 
confining behavior of the color-Coulomb potential, which is necessary condition for color confinement in Coulomb gauge QCD [8]. The transverse gluon propagator is expected to be suppressed in the IR region due to the proximity of the Gribov region [10].

\section{Simulation results: transverse gluon propagator}
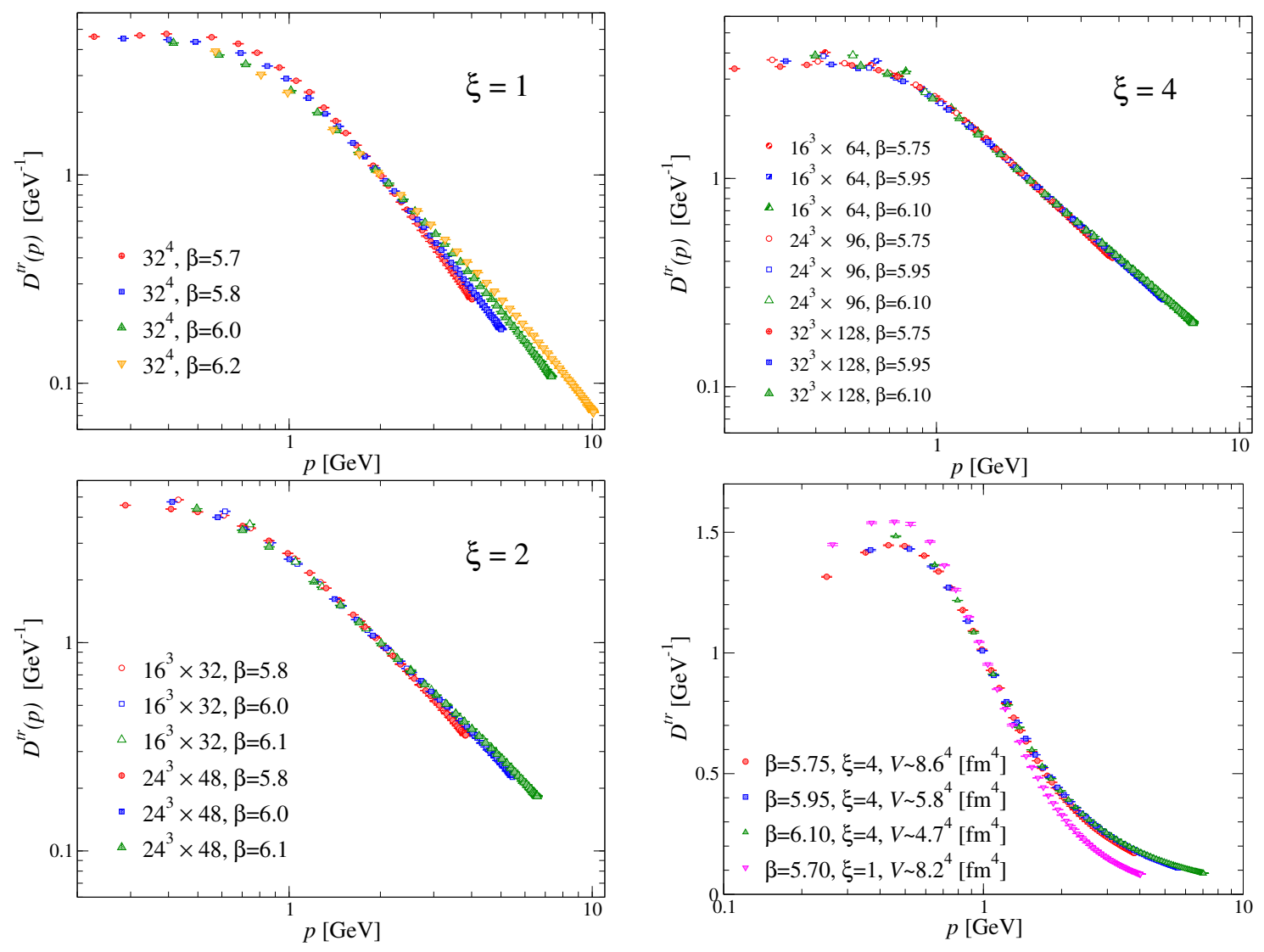

Figure 1: The equal-time transverse gluon propagator on the isotropic lattice (top left), on the anisotropic lattices with $\xi=2$ (bottom left), and with $\xi=4$ (top right). The results for the isotropic lattice and the anisotropic lattice with $\xi=4$ on large lattice volume are drawn together in one figure for direct comparison (bottom right). The cone cut and the cylinder cut are applied and the propagator is renormalized to unity at $p=2[\mathrm{GeV}]$.

The top left panel of Fig. 1 shows the transverse gluon propagator on the isotropic lattice at various lattice couplings, $\beta=5.7,5.8,6.0,6.2$. The cone cut and the cylinder cut are applied [20] and the propagator is renormalized such that $D^{t r}(|\vec{p}|=2[\mathrm{GeV}])=1$. We see that the data points at different lattice couplings cross at the renormalization point $|\vec{p}|=2[\mathrm{GeV}]$ and deviate from each other both at small and large momenta, as has been observed in [14].

The simulation results on the anisotropic lattice are drawn in the left bottom $(\xi=2)$ and the right top $(\xi=4)$ panel of Fig. 1 . On the anisotropic lattice with $\xi=2$, scaling violation becomes moderate compared to the isotropic result, although small deviations among the data points for different lattice couplings can be seen. Further increase of $\xi$ leads to a nice scaling behavior and 
the data points for $\xi=4$ almost fall on top of one curve, indicating that the equal-time gluon propagator is multiplicatively renormalizable in the continuum limit (or in the Hamiltonian limit $\xi \rightarrow \infty)$. Accordingly, our results on the anisotropic lattice support our expectation that scaling violation observed in the equal-time transverse gluon propagator disappears in the limit $\xi \rightarrow \infty$.

In the right bottom panel of Fig. 1, the instantaneous transverse gluon propagator on the spatial lattice extent $L=48$ is plotted both for the isotropic lattice and the anisotropic lattice with $\xi=4$. We observe that the propagator has a maximum at $p=0.4 \sim 0.5[\mathrm{GeV}]$ irrespective of the lattice coupling and the anisotropy, and it decreases with the momentum in the IR region.

\section{Simulation results: time-time component of the gluon propagator}
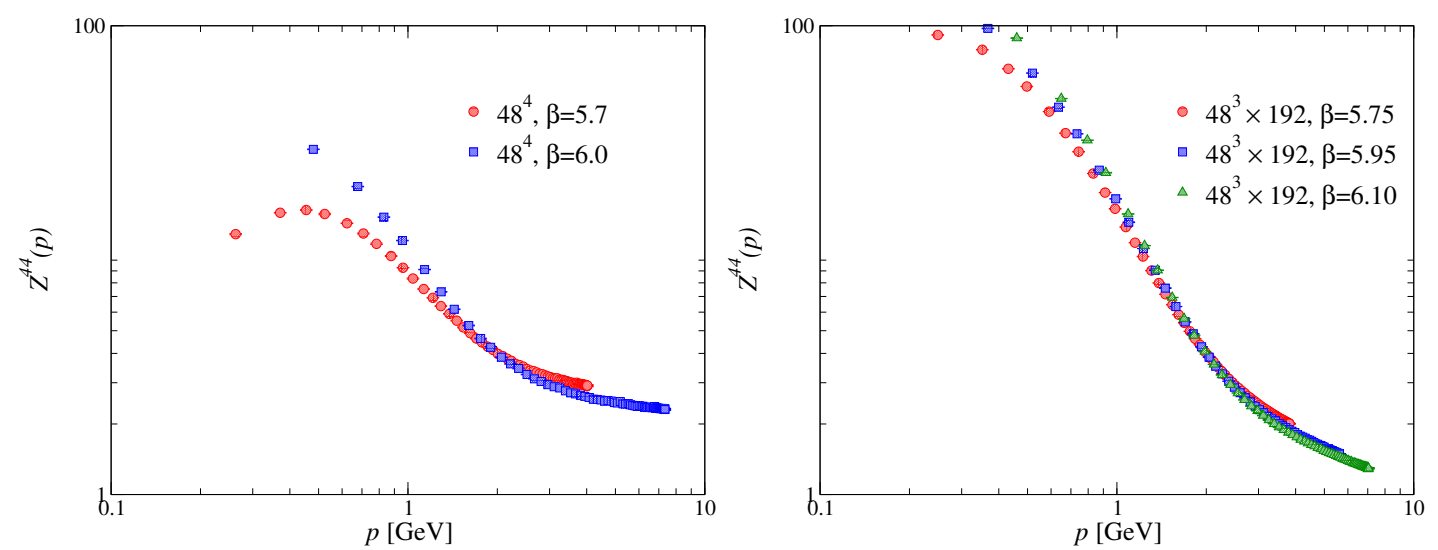

Figure 2: The dressing function of the time-time component of the gluon propagator on the isotropic lattice (left), and on the anisotropic lattice with $\xi=4$ (right). The cone cut and the cylinder cut are applied and the dressing function is renormalized to unity at $p=2[\mathrm{GeV}]$.

The dressing function of the time-time component of the gluon propagator is shown in Fig. 2 for the isotropic lattice (left panel) and the anisotropic lattice with $\xi=4$ (right panel). On the isotropic lattice, $Z^{44}(|\vec{p}|)$ shows scaling violation and the deviation of the two curve is pronounce in the IR region. Although the Gribov-Zwanziger scenario predicts that the temporal gluon propagator diverges stronger than the simple pole $1 /|\vec{p}|^{2}$, the numerical result shows that it bends down at small momenta for $\beta=5.7$.

On the anisotropic lattice, the dressing function shows a much better scaling behavior than that on the isotropic lattice. Although the small deviation can be seen both in the IR and ultraviolet region, one can expect that the scaling behavior is completely recovered in the Hamiltonian limit. Moreover, we find that the IR behavior of $Z^{44}$ on the anisotropic lattice is completely different from that on the isotropic lattice. For the isotropic case, we see that the dressing function bends down at small momenta at $\beta=5.7$. By contrast, $Z^{44}$ continues to rise with decreasing the momentum even for the coarsest lattice data $(\beta=5.75)$, and $Z^{44}$ at available smallest momentum for the anisotropic case is about 10 times larger than that for the isotropic case. We note that the spatial lattice spacing for $(\xi, \beta)=(4,5.75)$ is larger than that for $(\xi, \beta)=(1,5.70)$. This implies that $Z^{44}$ is very sensitive to the disretization effects, and taking the Hamiltonian limit is crucial to cure scaling violation for the temporal gluon propagator and to explore the IR divergent behavior in Coulomb gauge QCD. 


\section{Summary and conclusion}

We calculate the transverse and time-time components of the equal-time gluon propagator both on the isotropic and the anisotropic lattices. We find that scaling violation observed on the isotropic lattice is drastically reduced by calculating the propagator on the anisotropic lattices, i.e., by getting close to the Hamiltonian limit. In the IR region, the transverse gluon propagator is strongly suppressed and shows the turnover at about $500[\mathrm{MeV}]$. The time-time gluon propagator on the anisotropic lattice is much more enhanced in the IR region compared to that on the isotropic lattice.

\section{Acknowledgements}

The simulation was performed on NEC SX-8R at RCNP, and NEC SX-9 at CMC, Osaka University. We appreciate the warm hospitality and support of the RCNP administrators. Y. N. is supported by Grant-in-Aid for JSPS Fellows from Monbu-kagakusyo.

\section{References}

[1] D. Zwanziger, Nucl. Phys. B412, 657 (1994).

[2] J. Greensite, S. Olejnik and D. Zwanziger, JHEP 05, 070 (2005), [hep-lat/0407032].

[3] Y. Nakagawa, A. Nakamura, T. Saito and H. Toki, Phys. Rev. D75, 014508 (2007), [hep-lat/0702002].

[4] J. Greensite and S. Olejnik, Phys. Rev. D67, 094503 (2003), [hep-lat/0302018].

[5] A. Nakamura and T. Saito, Prog. Theor. Phys. 115, 189 (2006), [hep-lat/0512042].

[6] Y. Nakagawa, A. Nakamura, T. Saito, H. Toki and D. Zwanziger, Phys. Rev. D73, 094504 (2006), [hep-lat/0603010].

[7] Y. Nakagawa, A. Nakamura, T. Saito and H. Toki, Phys. Rev. D77, 034015 (2008), [arXiv:0802.0239 [hep-lat]].

[8] D. Zwanziger, Phys. Rev. Lett. 90, 102001 (2003), [hep-lat/0209105].

[9] A. Voigt, E. M. Ilgenfritz, M. Muller-Preussker and A. Sternbeck, Phys. Rev. D78, 014501 (2008), [0803.2307].

[10] D. Zwanziger, Nucl. Phys. B364, 127 (1991).

[11] A. Cucchieri and D. Zwanziger, Phys. Rev. D 65, 014001 (2001).

[12] K. Langfeld and L. Moyaerts, Phys. Rev. D70, 074507 (2004), [hep-lat/0406024].

[13] G. Burgio, M. Quandt and H. Reinhardt, Phys. Rev. Lett. 102, 032002 (2009), [0807.3291].

[14] Y. Nakagawa et al., Phys. Rev. D79, 114504 (2009), [0902.4321].

[15] T. R. Klassen, Nucl. Phys. B533, 557 (1998), [hep-lat/9803010].

[16] CP-PACS, Y. Namekawa et al., Phys. Rev. D64, 074507 (2001), [hep-lat/0105012].

[17] H. Matsufuru, T. Onogi and T. Umeda, Phys. Rev. D64, 114503 (2001), [hep-lat/0107001].

[18] S. Necco and R. Sommer, Nucl. Phys. B622, 328 (2002), [hep-lat/0108008].

[19] C. T. H. Davies et al., Phys. Rev. D37, 1581 (1988).

[20] D. B. Leinweber, J. I. Skullerud, A. G. Williams and C. Parrinello, Phys. Rev. D 60, 094507 (1999). 